\title{
SEROPREVALENCIA DEL Toxoplasma gondii EN BORREGAS DE UNA EMPRESA GANADERA DE LA SIERRA CENTRAL
}

\author{
Patricia Caldas J. ${ }^{1}$, Amanda Chávez V. ${ }^{2,3}$ y Eva Casas A. ${ }^{2}$
}

\section{AbSTRACT}

Toxoplasma gondii is an intracellular parasite of worldwide distribution that causes reproductive disorders in animals and man. Warm-blooded animals constitute the intermediate hosts, get tissue cysts and disseminate the disease when contaminate the pasture with feces containing oocysts. Domestic and wild feline are the definitive hosts. The objective of the study was to determine the seroprevalence of $T$. gondii in ewes belonging to a commercial farm located in the Central Highlands of Junín, Peru, using the Indirect Immunofluorescent Antibody Test (IFAT). A total of 387 sera were collected from ewes of various ages at shearing time in March 2002. The detected seroprevalence was $65.9 \pm 4.7 \%$ (255/387). Seroprevalence increased according to age $(\mathrm{p}<0.05)$.

Key words: Toxoplasmosis, Toxoplasma gondii, IFA, sheep, ewe

\section{RESUMEN}

El Toxoplasma gondii es un parásito intracelular de distribución universal, causante de problemas reproductivos en animales y el hombre. Los animales de sangre caliente constituyen los hospederos intermediarios, presentan quistes tisulares y diseminan la enfermedad al contaminar las pasturas con heces conteniendo ooquistes. Los felinos domésticos y silvestres son los hospederos definitivos. El objetivo del estudio fue determinar la seroprevalencia del $T$. gondii en borregas en edad reproductiva de una empresa ganadera de la Sierra Central del departamento de Junín, mediante la prueba de Inmunofluorescencia Indirecta (IFI). Se evaluaron 387 sueros de borregas de diferentes edades durante la esquila en marzo del 2002. Se halló una seroprevalencia a $T$. gondii de $65.9 \pm 4.7 \%$ (255/387). La seroprevalencia se incrementó con la edad de las borregas $(\mathrm{p}<0.05)$.

Palabras clave: Toxoplasmosis, Toxoplasma gondii, IFI, ovino, borrega

\footnotetext{
${ }^{1}$ Práctica privada

${ }^{2}$ Laboratorio de Microbiología y Parasitología Veterinaria, FMV-UNMSM

${ }^{3}$ E-mail:a_chavez_g@hotmail.com
} 


\section{INTRODUCCIÓN}

En el Perú existen 14 millones de ovinos, parte de los cuales se encuentran en empresas ganaderas donde la crianza es en forma extensiva. Es así, que en el departamento de Junín se encuentra una de las empresas pecuarias con mayor población ganadera, la Sociedad Agrícola de Interés Social (SAIS) Túpac Amaru, la cual cuenta con 120,000 cabezas de ovinos de raza Junín (Ministerio de Agricultura, 2001).

La toxoplasmosis es una de las principales enfermedades causantes de aborto en pequeños rumiantes, cuyo agente es el Toxoplasma gondii, parásito intracelular, de ciclo biológico complejo, que tiene por hospedero definitivo a los felinos silvestres y domésticos y como hospederos intermediarios a gran número de mamíferos y aves (Luzón et al., 1997a). En el ganado ovino ocasiona problemas reproductivos como muerte fetal, momificación, aborto y esterilidad (Rojas, 1990), trayendo como consecuencia pérdidas económicas por la disminución en la producción de lana y por pérdida de peso de los animales. Este problema es alarmante en países como Nueva Zelanda, Australia, Inglaterra, Noruega y Estados Unidos, donde hasta los años '80, el T. gondii ha sido identificado como agente causal de abortos en el $14 \%$ de los rebaños con problemas reproductivos, con una prevalencia media por rebaño de $12 \%$ de ovejas abortadas, lo que implica una incidencia anual de $1.7 \%$ de ovejas afectadas (Luzón et al., 1997b). En el Reino Unido se reportó que el $22 \%$ de los rebaños estaban afectados con toxoplamosis en 1992 (Stubbings, 1995). Asimismo, existen reportes de seroprevalencias elevadas en borregas a T. gondii en León, España (64\%) y en Suecia $(55 \%)$ con la prueba de inmunofluorescencia indirecta (Luzón et al., 1997b).

La toxoplasmosis es, además, un problema en salud pública, ya que el hombre se infecta al ingerir quistes tisulares contenidos en carnes poco cocidas procedentes de animales con quistes de toxoplasma. Así mismo, es una enfermedad oportunista que afecta a personas inmunosuprimidas con el Síndrome de Inmunodeficiencia Adquirida (SIDA) o con tratamiento de quimioterapia contra el cáncer (Acha y Szyfres, 1996).

Estudios sobre prevalencia de toxoplasmosis en el Perú indican que la infección está presente en el $40 \%$ de los ovinos (Leguía et al., 1986; Rojas, 1990), 58\% de caprinos (Vidal, 1990) y entre el 24 y $56 \%$ de alpacas (Leguía et al., 1987; Góngora, 1992; Gómez et al., 2003). Sin embargo, no existe información actualizada de este tipo de infección en ganaderías ovinas, de allí que se planteó el presente estudio para determinar la seroprevalencia del Toxoplasma gondii en borregas de una empresa ganadera de la Sierra Central del país.

\section{Materiales y Métodos}

\section{Localización y animales}

El presente estudio se llevó a cabo en la empresa ganadera Sociedad Agrícola de Interés Social Túpac Amaru, ubicada en las provincias de Jauja, Junín, Tarma y Yauli, del departamento de Junín, entre 3,500 y 4,700 msnm, y con una extensión de 180,000 ha. La zona presenta una época de seca y una de lluvias. La precipitación varía entre 600 a $900 \mathrm{~mm}$ anuales, con mayor intensidad en los meses de febrero y marzo. Las características botánicas de las praderas son en base a las asociaciones de pastos naturales (Alencastre, 1997).

Se colectaron muestras de sangre de borregas de la raza Junín en edad reproductiva de las siete unidades de producción del centro ganadero. Las muestras se tomaron entre marzo y junio del 2002, durante la temporada de esquila. Los sueros resultantes se almacenaron en congelación a $-20{ }^{\circ} \mathrm{C}$ hasta su análisis en el Laboratorio de Parasitología 
Cuadro 1. Seroprevalencia del Toxoplasma gondii en borregas Junín en edad reproductiva de la empresa ganadera Tupac Amaru (marzo-junio, 2002)

\begin{tabular}{|c|c|c|c|c|c|}
\hline & Muestras procesadas & Animales seropositivos & \multirow{2}{*}{$\%$} & \multirow{2}{*}{ \pm} & \multirow{2}{*}{ IC } \\
\hline & (n) & $\mathrm{n}$ & & & \\
\hline \multicolumn{6}{|c|}{ Unidad de producción } \\
\hline Pachacayo & 64 & 35 & 54.7 & \pm & 12.2 \\
\hline Atocsaico & 64 & 42 & 65.6 & \pm & 11.6 \\
\hline Casaracra & 69 & 61 & 88.4 & \pm & 7.6 \\
\hline Cónsac & 86 & 52 & 60.5 & \pm & 10.3 \\
\hline Pucará & 43 & 25 & 58.1 & \pm & 14.8 \\
\hline Cochas & 41 & 28 & 68.3 & \pm & 14.2 \\
\hline Quiulla & 20 & 12 & 60.0 & \pm & 21.5 \\
\hline \multicolumn{6}{|l|}{ Edad (en años) } \\
\hline $2-3$ & 98 & 54 & 55.1 & \pm & 9.9 \\
\hline $3-4$ & 138 & 91 & 66.0 & \pm & 7.9 \\
\hline$\geq 4$ & 151 & 110 & 72.9 & \pm & 7.1 \\
\hline Total & 387 & 255 & 65.9 & \pm & 4.7 \\
\hline
\end{tabular}

${ }^{1}$ Intervalo de confianza del $95 \%$

de la Facultad de Medicina Veterinaria de la Universidad Nacional Mayor de San Marcos.

El tamaño de muestra se calculó con la fórmula "para estimar una proporción" (Daniel, 1996), usando una proporción de 0.5 debido a que no había datos de prevalencia previos en la empresa ganadera. El número mínimo de muestras resultante fue de 385 borregas. Se estratificó el número de borregas entre las siete unidades de producción en base al tamaño poblacional de cada unidad.

\section{Análisis serológico}

Los sueros fueron analizados mediante la prueba de inmunofluorescencia indirecta (IFI) para la detección de anticuerpos antiToxoplasma gondii. El suero de la muestra fue diluido en 1:50 utilizando el antígeno (taquizoitos) proporcionado por la Universidad Complutense de Madrid (España) y usando el conjugado anti-ovino IgG de proceden- cia comercial (SIGMA) (Innes y EstebanRedondo, 1997).

\section{Análisis estadístico}

Los resultados se expresaron en porcentajes de positividad a $T$. gondii con sus respectivos intervalos de confianza del $95 \%$. Se realizó el análisis de regresión logística, con el fin de determinar la posible asociación entre la infección de Toxoplasma gondii en borregas con las unidades de producción y la edad de las borregas.

\section{Resultados}

El Cuadro 1 muestra la seroprevalencia del Toxoplasma gondii hallada en 387 borregas de la empresa ganadera bajo estudio. Se encontró 255 animales positivos, lo que implica una seroprevalencia de $65.9 \pm 4.7 \%$, con una variación entre unidades de produc- 
ción de 54.7 a $88.4 \%$. El análisis de regresión logística indicó que existe una mayor probabilidad de encontrar animales positivos en la unidad de producción Casaracra (0.0022) que en el resto de las unidades de producción $(\mathrm{p}<0.05)$.

Los resultados de la seroprevalencia, según el grupo etáreo, indicaron que la frecuencia de borregas seropositivas se incrementó con la edad (Cuadro 1).

\section{Discusión}

Se tiene conocimiento de dos estudios previos realizados en la Sierra Central del Perú donde se determinó la seroprevalencia del Toxoplasma gondii en borregas. La primera se hizo en la SAIS Pachacútec encontrando una prevalencia de 42\% (35/94) (Leguía et al., 1984) y la segunda en la SAIS Cahuide, con una seroprevalencia del 40\% (48/120), (Leguía et al., 1986), utilizándose la prueba de hemaglutinación indirecta en ambos casos. Estos resultados son inferiores al encontrado en el presente estudio, debido, probablemente, a la mayor sensibilidad de la prueba de inmufluorescencia indirecta (Gorman et al., 1999) y del tamaño muestral. Otros estudios realizados en borregas de los departamentos de Ancash, Junín y Puno, empleando la prueba de hemaglutinación indirecta reportan seroprevalencias similares al presente estudio (68.4\% en época de lluvias y 55.8\% en época de seca) (Ameghino, 1988). Se espera una mayor prevalencia en época de lluvia, dado que en esa época hay una mayor supervivencia del ooquiste en los pastos, y por lo tanto, una mayor probabilidad de infección (Rojas, 1990).

Las diferencias halladas entre unidades de producción podrían deberse a factores tales como la influencia de la cercanía a poblados, ya que se encuentra cerca a la carretera Oroya-Tarma, por lo que existiría una mayor posibilidad de contacto con los gatos domésticos; en cambio, la unidad de Pachacayo, que tiene el menor nivel de prevalencia, está alejada de la carretera Oroya-Jauja. Así mismo, también se ha reportado diferencias en las tasas de seroprevalencia entre animales que viven a nivel del mar, con aquellas que viven en las alturas (Acha y Szyfres, 1986).

Estudios previos han demostrado que la presencia de felinos domésticos y silvestres constituyen un factor de riesgo importante para la contaminación del medio ambiente con formas infectivas del parásito (Rojas, 1990; Gómez et al., 2003). En las unidades de Cochas, Cónsac y Atocsaico se ha reportado la presencia de pumas. Otros factores que también favorecerían la infección por $T$. gondii sería la disminución de la inmunidad del hospedero por el estrés del manejo durante las faenas de esquila, dosificación, baños antiparasitarios y sobrecarga en las zonas de pastoreo; además que algunas de estas faenas se realizan cerca de zonas pobladas y consecuentemente, cerca de los hospederos definitivos (Leguía, 1999). Otro factor sería el estrés provocado por el parto y el empadre, que ocasionan una inmunosupresión fisiológica (Rojas et al., 1989).

El incremento de la seroprevalencia con la edad de los animales encontrado en el presente estudio fue similar a los reportes de Leguía et al. (1984, 1986).

La infección se produciría por ingestión de alimentos contaminados con ooquistes esporulados a partir del momento del destete, aunque se reportan mayores tasas de infección en animales adultos, ya que tienen mayores probabilidades de sufrir la infección. La primoinfección en edades tempranas logra ser fuente de inmunidad protectora que impediría la infección del feto en futuras gestaciones, y por lo tanto se evitaría la presentación de abortos. La prueba de regresión logística demostró que las borregas mayores de 4 años tienen mayor riesgo en adquirir la infección $(\mathrm{p}<0.05)$, confirmando reportes previos que indican el mayor riesgo de exposición para contraer toxoplasmosis a medida que se incrementa la edad en los animales (Dubey y Lappin, 2000). 
La seroprevalencia a Toxoplasma gondii hallada en el presente estudio fue superior a otras especies. Así, se reporta $14.8 \%$ en vicuñas de Puno (Pastor, 2002) y $21 \%$ en alpacas de la unidad de producción de Cochas (Poma, 2003). Esto se debe a que estos animales viven en alturas superiores a los 4,000 msnm, mientras que los ovinos no sobrepasan los 3,800 msnm. Los animales se encuentran más alejados del contacto humano y se cumple la relación que a mayor altura sobre el nivel del mar, es menor la probabilidad del contacto con esta coccidia.

La alta prevalencia obtenida en el estudio indicaría la presencia de gran cantidad de formas quísticas de $T$. gondii en los tejidos de las borregas, por lo que se recomienda una adecuada cocción de la carne de estos animales para su consumo.

\section{Literatura Citada}

1. Acha, P.; B. Szyfres. 1986. Zoonosis y enfermedades transmisibles comunes al hombre y a los animales. $2^{\mathrm{a}}$ ed. $\mathrm{p}$ 646657. Publicación Científica 503. OPS. Washington, EEUU.

2. Alencastre, R. 1997. Producción de ovinos. p 21-57. Ed. A \& R. Panamericana. Puno, Perú.

3. Ameghino, E. 1988. Avances sobre investigación en salud animal - Ovinos. Bol. Div. IVITA-UNMSM. 21: 50.

4. Daniel, W. 1996. Bioestadística base para el análisis de las ciencias de la salud. $5^{\text {a }}$ ed. p 205-207. Ed. Limusa. México.

5. Dubey, J.P.; M.R. Lappin. 2000. Toxoplasmosis y neosporosis. En: Enfermedades infecciosas en el perro y gato. $2^{a}$ ed. p 542-553. Ed. McGraw-Hill Interamericana. México.

6. Gómez, F.; A. Chávez; E. Casas; E. Serrano; O. Cárdenas. 2003. Determinación de la seroprevalencia de toxoplasmosis en alpacas y llamas en la Estación Experimental INIA-Puno. Rev. Inv. Vet. Perú 14: 49-53.
7. Góngora, M. 1992. Prevalencia de anticuerpos contra Toxoplasma gondii en las comunidades alpaqueras de Vilcallamas, Bajo Llallahua, Huanacayama, Llusta. Tesis de Médico Veterinario y Zootecnista. Universidad Nacional del Altiplano. Puno. 47 p.

8. Gorman, T.; J.P. Aranciba; M. Lorca; D. Hird; H. Alcaino. 1999. Seroprevalence of Toxoplasma gondii infection in sheep and alpacas (Lama pacos) in Chile. Prev. Vet. Med. 40: 143149.

9. Innes, E.A.; M.I. Esteban-Redondo. 1997. Diagnóstico. En: Tratado de patología y producción ovina. L. Ortega (ed). Cap. 4. Ed. Luzán. Madrid.

10. Leguía, G.; C. Guerrero; M. Rojas; H. Samamé. 1984. Prevalencia de Toxoplasma gondii en ovinos de la sierra central. VII Reunión Científica Anual Asociación Peruana de Producción Animal (APPA). Lima, Perú.

11. Leguía, G.; C. Guerrero; P. Dionisio. 1986. Prevalencia de Toxoplasma gondii en borregas. IX Reunión Científica Anual Asociación Peruana de Producción Animal (APPA). Lima, Perú.

12. Leguía, G.; H. Samamé; C. Guerrero; M. Rojas; A. Nuñez. 1987. Prevalencia de anticuerpos contra Toxoplasma gondii en alpacas. MV Rev. Cienc. Vet. 3: 19-21.

13. Leguía, G. 1999. Enfermedades parasitarias de camélidos sudamericanos. p 3134. Ed. de Mar. Lima, Perú.

14. Luzón, M.; A. Alonso; G.A. Quintanilla. 1997a. Etiología, biología y epidemiología. En: Tratado de patología y producción ovina. L. Ortega (ed). Cap. 1. Ed. Luzán. Madrid.

15. Luzón, M.; G. Miro; G.A. Quintanilla. 1997b. Epidemiología. En: Tratado de patología y producción ovina. L. Ortega (ed). Cap. 4. Ed. Luzán. Madrid.

16. Ministerio de Agricultura. 2001. Atlas departamental del Perú 2003, Junín/ Huancavelica. p 39-42. Tomo 10. Ed. Peisa. Lima, Perú. 
17. Pastor, J. 2002. Seroprevalencia de Toxoplasma gondii en vicuñas de Puno. Tesis de Médico Veterinario. Facultad de Medicina Veterinaria, Univ. Nacional Mayor de San Marcos. Lima. 43 p.

18. Poma, E. 2003. Seroprevalencia de anticuerpos contra Toxoplasma gondii en alpacas (Lama pacos) de la Unidad de Producción de Cochas de la SAIS Túpac Amaru. Tesis de Médico Veterinario. Facultad de Medicina Veterinaria, Univ. Nacional Mayor de San Marcos. Lima. $41 \mathrm{p}$.

19. Rojas, M.; I. Lobato; M. Montalvo. 1989. Prevalencia de Toxoplasma gondii en camélidos sudamericanos. XII Reunión Científica Anual Asociación
Peruana de Producción Animal (APPA). Lima, Perú.

20. Rojas, M. 1990. Parasitismo de los rumiantes domésticos. Terapia, prevención y modelo para su aprendizaje. p 326-333. Ed. Mijosa. Lima, Perú.

21. Stubbings, L. 1995. Calculating the cost of toxoplasmosis to the sheep farmer. Proc. Sheep Veterinary Society 19: 1-3. Washington.

22. Vidal, L. 1990. Prevalencia de anticuerpos contra Toxoplasma gondii en cabras de la provincia de Lima. Tesis de Médico Veterinario. Facultad de Medicina Veterinaria, Univ. Nacional Mayor de San Marcos, Lima. 41 p. 